\title{
Radioterapia adyuvante en el cáncer de mama
}

\author{
H. Marsiglia, C. Widakowich, R. Rivera
}

\section{Epidemiología del cáncer de mama}

\section{Datos epidemiológicos sobre el cáncer de mama}

El cáncer de mama es el cáncer más frecuente de la mujer occidental, con 11.000 muertes por año solamente en Francia. La incidencia de esta patología aumenta con la edad, llegando su máximo alrededor de los 70 años.

Incidencia (por 100.000 personas) del cáncer de mama en Francia de 1984 a 1995 (de VAthaire 1996)

\begin{tabular}{cc}
\hline Age & $\%$ \\
\hline $35-39$ & 63.3 \\
$40-44$ & 119.7 \\
$45-49$ & 187.3 \\
$50-54$ & 177.3 \\
$55-59$ & 182.8 \\
$60-64$ & 211.3 \\
$65-69$ & 220.0 \\
$70-74$ & 231.1 \\
$75+$ & 220.4 \\
Conjunto & 89.2 \\
\hline
\end{tabular}

Se espera un aumento de los casos diagnosticados en Europa en el decenio 2000-2010 en la mujer de la tercera edad, en razón al aumento constante de la esperanza de vida, más marcado en la mujer que en el hombre (Kurtz 2002).

Hoy en día el riesgo de desarrollar un cáncer de mama antes de los 90 años es del $11 \%$ ( 1 de cada 9 mujeres desarrollara un cáncer de mama antes de los 90 años). El cáncer de mama representa entonces, un problema de salud pública importante, sobretodo en el grupo en el que el aumento de la esperanza de vida de las mujeres, coincide con la alta tasa de incidencia de este tumor.

Institut Gustave-Roussy, Villejuif (Francia)
Dos cosas podemos decir con certeza: primero, que la acción terapéutica debe ser considerada como un problema médico-económico de primer orden (Balducci 1997). Y segundo, que se debe detectar lo más precozmente posible el cáncer de mama. En los últimos años, el 50\% de los tumores han sido diagnosticados en un tamaño menor a los $2 \mathrm{~cm}$, y el diagnóstico de las lesiones no palpables ha representado el $40 \%$ de los cánceres detectados (Fig. 1). En este orden, la detección de los carcinoma in situ aumenta también, y puede representar hoy en día el $25 \%$ de los tumores diagnosticados.

De esta manera, las estrategias terapéuticas del tipo cirugía conservadora y los tratamientos adyuvantes están directamente relacionados con el aumento de la detección de los estadios precoces. El acrecentamiento de la incidencia del cáncer de mama en la mujer de cierta edad nos obliga a redirigir los protoclos clásicos de la radioterapia, modificándolos por esquemas de administración más cortos, asegurando un buen resultado estético, junto a un máximo control local en relación a los años de supervivencia.

\section{Standard de la radioterapia en el carcinoma in situ}

El carcinoma canalicular in situ (CCIS), representaba el $5 \%$ de los cánceres de mamas ante el diagnóstico mamografico, y hoy en día, esta tasa es del 20-30\%. El in situ en este

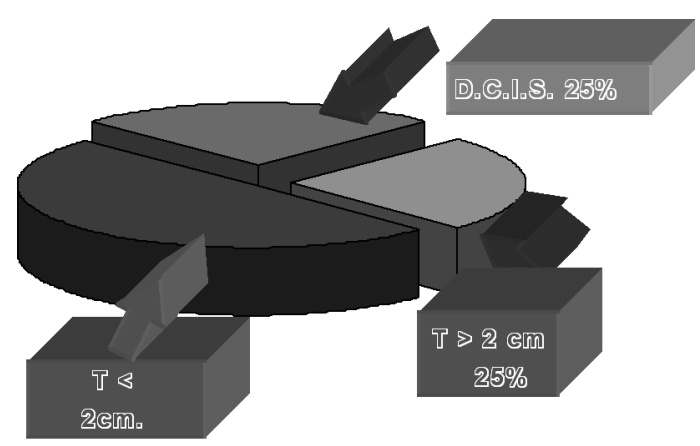

Fig. 1. 


\section{H. Marsiglia y cols.}

NSABP B-17

EORTC $10853^{2}$

Cutuli ${ }^{3}$

\begin{tabular}{|c|c|c|c|c|c|c|c|}
\hline Recidivas & $\begin{array}{l}\text { Seguidos } \\
\text { (en meses) }\end{array}$ & $\begin{array}{c}\mathrm{Ct} \\
(\mathrm{N}=403)\end{array}$ & $\begin{array}{c}\mathrm{CT}+\mathrm{RT} \\
(\mathrm{N}=411)\end{array}$ & $\begin{array}{c}\mathrm{CT} \\
(\mathrm{N}=500)\end{array}$ & $\begin{array}{c}C T+R T \\
(\mathrm{~N}=502)\end{array}$ & $\begin{array}{c}C T \\
(\mathrm{~N}=190)\end{array}$ & $\begin{array}{c}\mathrm{CT}+\mathrm{RT} \\
(\mathrm{N}=515)\end{array}$ \\
\hline \multirow[t]{3}{*}{ Total } & 51 & - & - & $16,6^{7}$ & $10,5^{7}$ & - & - \\
\hline & 90 & - & - & - & - & $31^{10}$ & $13^{10}$ \\
\hline & 129 & $31,7^{4}$ & $15,7^{4}$ & - & - & - & - \\
\hline Rec. in situ (\%) & & $14,6^{5}$ & $8^{5}$ & $8,6^{8}$ & $5,7^{8}$ & 15 & 5 \\
\hline Rec. invasiva (\%) & & 17,16 & $7,7^{6}$ & $8^{9}$ & $4,8^{9}$ & 16 & 8 \\
\hline
\end{tabular}

'Fisher B et al, Sem Oncol, 2001; 28: 400-18. ${ }^{2} \mathrm{Bijker} \mathrm{N}$ et al, JCO, 2001; 19: 2263-71. ${ }^{3} \mathrm{Cutuli} \mathrm{B}$ et al, IJRO, 2002; 53: 868-79. ${ }^{4} \mathrm{p}<0.000005$. ${ }^{5} p=0.00001 .{ }^{6} p=0.001 .{ }^{7} p=0.005 .{ }^{8} p=0.06 .{ }^{9} p=0.04 .{ }^{10} p<0.0001$

caso, es una lesión de muy buen pronóstico con menos de un $25 \%$ de evolución metastásica, donde la mastectomía era el tratamiento de referencia en la década del 70, con una cura del $98 \%$ de los casos (Holland 1985, De Vita, 2001). En esta década presente, muchos autores han intentado tratar al cáncer in situ, con cirugía sin Rt externa, pero la tasa de recidiva local ha aumentado a una media de $25-35 \%$ a los 10 años. Con la finalidad de mejorar el control local, muchos autores propusieron la cirugía+Rt. (Fisher, 1993), cosa que ha bajado la tasa de recidivas locales a un $8-15 \%$ a los 10 años, en relación a la calidad de la exéresis quirúrgica, de la edad del paciente, el grado, y los sub-tipos histológicos.

Los trials randomizados (exclusivos para los CCIS) National Surgical Adjuvant Breast and Bowel Project B17 (NSABP B-17), y European Organization for Research and Treatment of Cancer (EORTC) 10853 han comparado la tumorectomía versus la tumorectomía + radioterapia (50 Gy en un booster), y un trabajo multicéntrico retrospectivo francés (Cutuli, 2002) confirmó los beneficios de la irradiación después de la tumorectomía (sin modificación de la sobrevida).

La mitad de las recidivas son recidivas in situ; la otra mitad son recidivas en progreso bajo la forma de carcinomas infiltrantes $y$, en este caso, podemos ver un desarrollo de metástasis de un 15 a 20\%, dando un pronóstico peyorativo.

\section{Perspectivas terapéuticas}

\section{Consenso de St. Gallen}

Las indicaciones standard sugieren una tumorectomía sin curaje axilar con radioterapia sistemica (50 Gy) en los pacientes portadores de tumores de una talla inferior a los 4 $\mathrm{cm}$, sin localización multifocal, y una mastectomía (seguida de una reconstrucción mamaria inmediata) si la talla es superior a los $4 \mathrm{~cm}$. o si hay localización multifocal.

\section{Recomendaciones}

En todos los casos, la terapéutica debe ser óptima, haciendo una mamografía pre-operatoria completa, una exéresis completa de la lesión con márgenes mayores a $1 \mathrm{~cm}$, análisis histológico preciso (talla, márgenes y grado), y una mamografía post-operatoria de control antes de comenzar la radioterapia, para verificar la ausencia de microcalcificaciones residuales. Se debe aumentar el control en el caso de ser pacientes en riesgo, como los son la mujer joven, una exéresis limítrofe, o el alto grado tumoral.

\section{Perspectivas terapéuticas en el carcinoma intracanalicular in situ}

Los trials randomizados de la actualidad, muestran que todos los subgrupos de pacientes (edad, talla, grado, márgenes portadores de un CCIS, beneficiados por la RT, disminuyen un $50 \%$ el riesgo de recidiva después de la cirugía, y particularmente las recidivas bajo la forma infiltrante.

Dentro de este panorama, se nos aparecen varias preguntas:

1. No podemos definir actualmente los factores pronósticos para predecir la recidiva local del tipo infiltrante. Los intentos en el campo de la prevención de recidivas intracanaliculares e infiltrantes de los CCIS siguen siendo hoy en día el objeto de numerosos estudios:

- Estudio que testea al tamoxifeno (NSABP B-24, Fisher 2001) mostrando que una mujer sobre 20 pueden esperar un beneficio por el tamoxifeno; una de 52 evitará una recidiva local invasiva y una de 128 evitará una recidiva local in situ. Inclusive, es necesario relativizar los resultados domboembólicos inducidos por el tamoxifeno.

- Estudio prospectivo NSABP B-35 randomizado en 3.000 pacientes post-menopáusicas con CCIS comparando el tamoxifeno versus el anastrazol, después de la tumorectomía + RT.;

- Proyecto prospectivo utilizando los inhibidores de la Cox-2 para la prevención de recidivas invasivas de los carcinomas canaliculares in situ de la mujer menor a 50 años. Fase III randomizado (después de la tumorectomía+ radioterapia $50 \mathrm{~Gy}$ ) celecoxib versus placebo (en curso de aprobación al IGR).

2. No existen Trials que controlen el efecto de la dosis de la radioterapia en los CCIS. La dosis standard de 50 Gy podría ser subestimada para el control local de los CCIS (Kurtz, 2002) debiendo estudiarse el efecto de una dosis complementaria. En este sentido, hay un estudio randomizado (Estudio EORTC dado a aprobación Breast cancer conserving therapy in DCIS) evaluando el impacto de un boost sobre el control local y la incidencia de recidivas invasivas y no invasivas. 1346 pacientes seran incluidos en dos grupos randomizados: 50 Gy versus $66 \mathrm{~Gy}(50+16)$.

3. Una tumorectomía sin radioterapia podría estar indicada en los pacientes de muy buen pronóstico (talla $<1 \mathrm{~cm}$, bajo grado, unifocal, margenes $\geq 1 \mathrm{~cm}$ ), aunque aquí no tenemos trabajos validados hasta la fecha. El score de Van Nuys (talla-margen-grado) podría ser uno de ellos, (23\% de 
comedonecrosis sobre un total de 93 pacientes de estas características), pero no ha sido aprobado como estudio prospectivo aun.

\section{Standard de la radioterapia en los carcinoma infiltrante}

\section{Tratamientos conservadores}

Los cánceres de mama pueden beneficiarse de un tratamiento conservador. En efecto, la asociación Rt+Ct es la estreategia más utilizada. Dentro de este caso, la cirugía ha mostrado una evolución remarcable.

La cirugía en el tratamiento del cáncer de mama ha estado vista como un arma eficaz pero agresiva, sobre todo desde el punto de vista psicológico. Las primeras intervenciones descritas por Halsted (Brenier 1967, Ravitch 1971) mostraban secuelas funcionales, como el brazo aumentado de tamaño (Chardot 1968, Arnulf 1971). La mastectomía radical modificada por Patey (Nemoto 1975, Cunsulo 1979, Maddox 1983) logró mejorar ciertos problemas funcionales (siempre hablando de las secuelas de miembro superior) pero hubo que esperar hasta el comienzo de los años 80 (Veronesi 1981, Fisher 1989, Fisher 1993), para ver la aparición de un tratamiento realmente conservador, y el advenimiento de protocolos de cirugía conservadora+radioterapia adyuvante, que llegaron a brindar resultados muy esperanzadores. En efecto, los resultados de los diferentes trials randomizados publicados a lo largo de estas décadas (Fischer 1989, Jacobson 1995, Arriagada 1996, Van Dongen 2000) confirman la equivalencia en términos de sobrevida global entre los pacientes tratados por cirugía radical no conservadora y aquellos que podían beneficiarse de un tratamiento conservador radio-quirúrgico

\section{Comentario de los trials mastectomía versus tratamiento conservador}

El límite superior del diámetro tumoral para autorizar un tratamiento conservador variaba entre los 2 y los $5 \mathrm{~cm}$. De la misma manera los trials diferían según la naturaleza de la cirugía conservadora y la quimioterapia utilizada. La radioterapia era administrada a 45 a 50 Gy en 4,5 a 5 semanales. La tasa de recidivas locales varía según los diferentes estudios, entre un 5 a $20 \%$ después del tratamiento conservador y un 3 a 12\% después de la mastectomía. La tasa de metástasis a distancia y la sobrevida no eran, sin embargo, tan diferentes según el tipo de tratamiento. Es decir, la presencia de una invasión ganglionar axilar no estaba asociada a una diferencia en la sobrevida entre los dos tipos de tratamientos. Tres grandes méta-análisis (EBCTCG, 1995, Winchester, 1997, Morris, 1997), confirmaron esta igualdad en la sobrevida. De igual manera, no existía una diferencia entre las tasas de recidivas contralaterales y los tumores homolaterales no carcinomatosos. Finalmente, estos resultados fueron el standard de los años 80: cirugía conservadora y radioterapia de la mama para los carcinomas menores a $5 \mathrm{~cm}(\mathrm{NIH}$ Consensus 1991).

\section{Trials randomizados comparando la cirugía conservadora} sola versus cirugía seguida de irradiación

Entonces, ¿̇podemos decir que la radioterapia es indispensable en caso de tratamiento de mama conservador? Los trials randomizados que respondieron a esta cuestión (Fischer 1995, Clark 1996, Liljergen 1999, Veronesi 2001), concluyeron todos que la ausencia de radioterapia post-operatoria, en el tratamiento conservador, elevaba el riesgo de recidivas a aproximadamente un $30 \%$, contra el $10 \%$ de la asociación radio-cirugía. De esta manera, la tumorectomía asociada al curetaje axilar, seguida de radioterapia externa, devino al comienzo de la década de los 90, el standard de los tratamientos conservadores de cáncer de mama localizados, no inflamatorios, menores a $5 \mathrm{~cm}$.

\section{Comentarios de los trials}

El estudio NASBP B-06 comprendía tres tratamientos a comparar: mastectomía, tumorectomía sola y tumorectomía seguida de radioterapia, incluyendo los pacientes T1, T2,

Trials randomizados comparando la mastectomía con un tratamiento conservador radio-quirúrgico

\begin{tabular}{lccccccc}
\hline Autor & Pub. & Año & Nro de Pac. & Resultados Mastect & Resultados Tum+RT & SG & Sobrevida (años) \\
\hline Fischer & NEJM & 1989 & 1851 & $3 \%$ & $10 \%$ & NS & 15 \\
Jacobson & NEJM & 1995 & 247 & $10 \%$ & $5 \%$ & NS & 10 \\
Arriagada & JCO & 1996 & 179 & $14 \%$ & $9 \%$ & NS & 15 \\
Van Dongen & JNCl & 2000 & 868 & $12 \%$ & $20 \%$ & NS & 10 \\
\hline
\end{tabular}

Trials randomizados comparando los tratamientos conservadores con o sin radioterapia

\begin{tabular}{|c|c|c|c|c|c|c|c|}
\hline Autor & Pub. & Año & Nro de Pac. & RL Tum & Rl Tum + RT & Sobrevida & Seguidos (años) \\
\hline Fisher & NEJM & 1995 & 1851 & $36 \%$ & $12 \%$ & NS & 15 \\
\hline Clark & $\mathrm{JNCl}$ & 1996 & 837 & $35 \%$ & $11 \%$ & NS & 4 \\
\hline Forrest & Lancet & 1996 & 585 & $24,5 \%$ & $5,8 \%$ & NS & 6 \\
\hline Liljergen & $\mathrm{JCO}$ & 1999 & 381 & $24 \%$ & $8,5 \%$ & NS & 10 \\
\hline Veronesi & Ann Oncol & 2001 & 579 & $23.5 \%$ & $5.8 \%$ & NS & 12 \\
\hline
\end{tabular}




\section{H. Marsiglia y cols.}

\begin{tabular}{lcccccccc} 
Autores & Pub. & Año & Nro. Pac. & RT + boost (Gy) & RL Boost - & RL Boost + & Sobrevida & Seguidos (en años) media \\
\hline Romestaing & JCO & 1997 & 1024 & 50 vs 50+10 & $4.5 \%$ & $3.6 \%$ & NS & 3.3 \\
Bartelink & NEJM & 2001 & 5318 & 50 vs 50+16 & $7.3 \%$ & $4,3 \%$ & NS & 5.1 \\
\hline
\end{tabular}

hasta $4 \mathrm{~cm}$, NO clínico. En este estudio, después de la tumorectomía, la RT reducía la tasa de recidivas de 36 a 12\% a 15 años $(p<0.001)$. Esta reducción era todavía más importante cuando había invasión ganglionar axilar (tasa de recidiva del $43 \%$ para los pacientes $\mathrm{N}+$ no irradiados, versus $6 \%$ y tasa de recidiva del $37 \%$ por los pacientes $N$ - no irradiados versus $12 \%$ ). Los pacientes con invasión ganglionar fueron todos tratados con una quimioterapia adyuvante, dando como resultado, el hecho de que el tratamiento sistémico no es suficiente para asegurar el control local de la enfermedad. En definitiva, estos trials demostraron que la RT después de la cirugía conservadora disminuía el riesgo de recidivas de $60-75 \%$ (per sin impacto sobre la sobrevida global). La RT adyuvante aparecía entonces, como el tratamiento standard después de la tumorectomía o cuadrantectomía+curetaje axilar.

\section{Trials randomizados comparando la eficacia en términos de recidiva local, de una irradiación a 50Gy, versus 50Gy asociados a un complemento sobre el sitio tumoral}

Los progresos recientes en materia de tratamiento conservador radio-quirúrgico del cancer de mama, se caracterizan por el interés de probar un aumento de la dosis sobre el sitio tumoral, valorando una diferencia significativa en términos de control local de la enfermedad (sin beneficio en la sobrevida). El complemento de irradiación es de 10 a $16 \mathrm{~Gy}$ sobre el lecho tumoral inicial, después de la irradiación del conjunto de la glándula mamaria a 50 Gy (Bartelink 2001, Romestaing 1997).

\section{Comentario de los trials}

El estudio randomizado l'EORTC 22881 (Bartelink 2001), compara el efecto de un complemento de 16Gy sobre el lecho tumoral y la tasa de recidiva local, dando un amplio beneficio en esta última. Esta diferencia es ampliamente significativa para los pacientes menores a los 40 años $(p=0.002)$, significativa para aquellos entre de 41 a 50 años $(p=0.02)$, cerca de un $p<0.05$ para los pacientes entre 51 y 60 años $(p=0.07)$ y no significativa para los pacientes mayores 60 años ( $p=0.11$, Fig. 2).

Si comparamos los resultados en un valor absoluto, podemos constatar sin embargo, que la eficacia de un boost de 16 Gy sobre el lecho tumoral permite disminuir en un factor cercano a 2 el riesgo de recidiva local tanto en mujeres menores de 40 años (19.5 versus 10,2\%) como en los mayores a 60 años (4 versus 2,5\%). El factor de la edad es altamente significativo, pues el riesgo de recidivas locales puede depender más de esta variable, que de la "ineficacia del boost".

El estudio de Lyon (Romestaing 1997), con 1.024 pacientes tratados por fumorectomía y curetaje axilar para los tumores $<$ a $3 \mathrm{~cm}$. Los pacientes recibían una irradiación durante 5 semanas en 20 fracciones, de $50 \mathrm{~Gy}$, seguidas o no

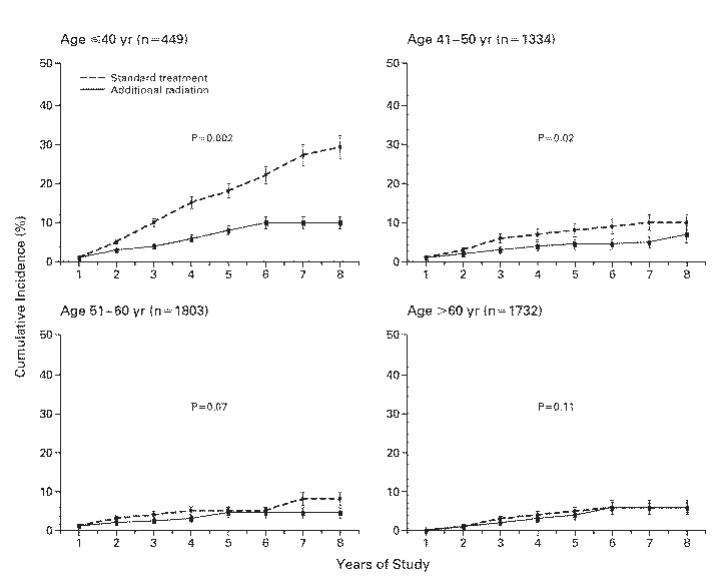

Fig. 2.

de un complemento por electrones de 10Gy, sobre el lecho tumoral. El seguimiento fue de una media de 3.3 años. Cuestión, la tasa de recidivas locales a 5 años sin boost fue del $4.5 \%$, y del $3.6 \%$ con boost ( $p=0,044)$.

En lo concerniente a la realización de un boost después de la resección completa de un cáncer de mama, no existen

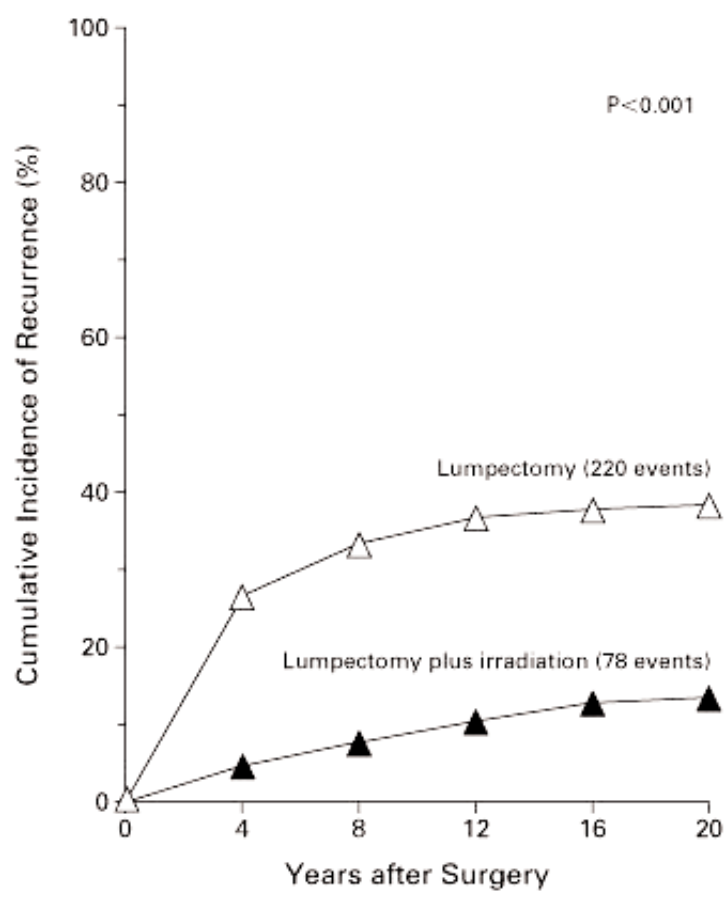

Fig. 3. Fisher. 


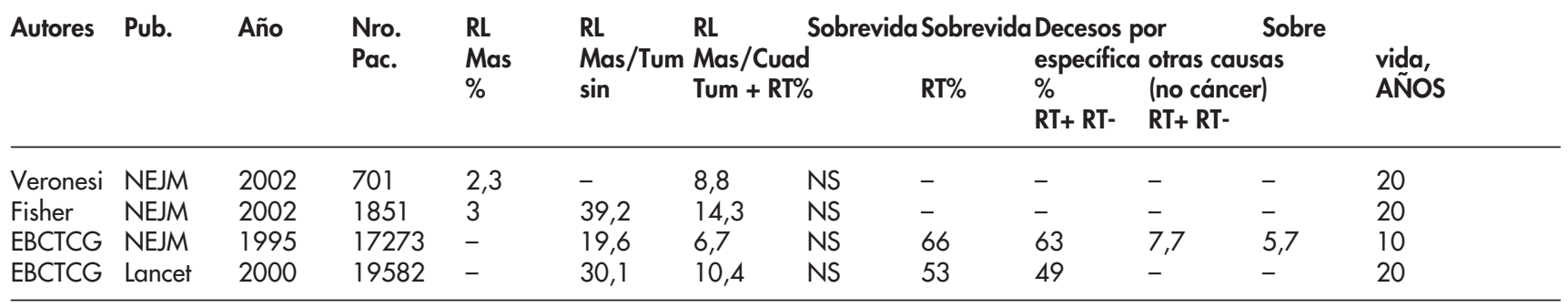

datos suficientes para proponer un boost de radioterapia externa (fotones, electrones) o por curiterapia. La calidad del resultado estético es controversial según la técnica, dependiendo del volumen mamario, de la localización tumoral, y de la calidad del centro medico.

Trials actualizados a largo plazo, mostrando el devenir de las recidivas locales, y su influencia en la sobrevida

Numerosos estudios retrospectivos muestran una eficacia muestran una eficacia de la radioterapia adyuvante, versus la mastectomia o la cirugía conservadora sola. Así lo demuestran la actualización de los trials históricos (Veronesi, Fisher 2002) y los meta-análisis de l'Early Breast Cancer Trialists Collaborative Group (EBCTCG) publicados en 1995 y 2000 .

\section{Comentarios de los trials}

Los trials de Veronesi y Fisher muestran bien que la tasa de recidiva local a largo plazo no es lineal. Admitimos que esa tasa progresa alrededor de un 1\% anual en la asociación radio-cirugía, y el triple con la cirugía sola (Fisher 2002, Fig. 3)

Los resultados a largo plazo entre mastectomía y cuadrantectomía con o sin RT externa, no mostraron variaciones con la sobrevida global (Fisher 2002, Veronesi, 2002).

\section{Trials demostrando la correlación entre recidiva local e influencia en la sobrevida}

Muchos autores han publicado que el aumento de la incidencia de las metástasis a distancia, se relaciona con la recidiva local, condicionando así la sobrevida (Fisher, 1991; Haffty, 1996; Fortin, 1999). El trabajo retrospectivo Koscielny (IJROBP, 1999), compara 3.679 pacientes tratados entre 1954 et 1975 en el Instituto Gustav-Roussy, mostrando que el plazo medio de aparición de metástasis aumenta de 5 a 15 años, cuando los pacientes presentaron recidiva local.

\section{Meta-análisis mostrando el impacto de la RT en la recidiva local y la sobrevida}

Los meta-análisis: Early Breast Cancer Trialists Collaborative Group (EBCTCG) de 1995 (Effects of radiotherapy and surgery in early breast cancer; NEJM) y 2000 (Favourable and unfavourable effects of long-term survival of radiotherapy for early breast cancer: an overview of the randomised trials; Lancet) volvieron a confirmar los beneficios de la RT en terminos de control local, y disminuyendo de un 30 a un

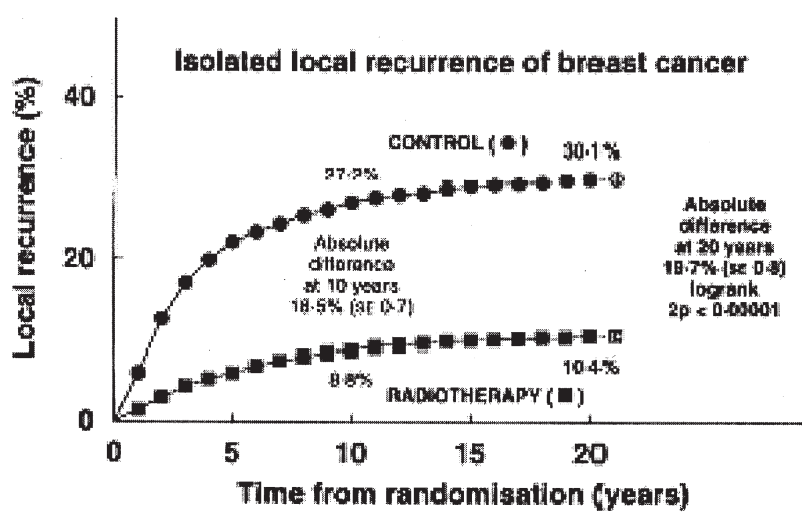

Fig. 4. EBCTCG 2000.

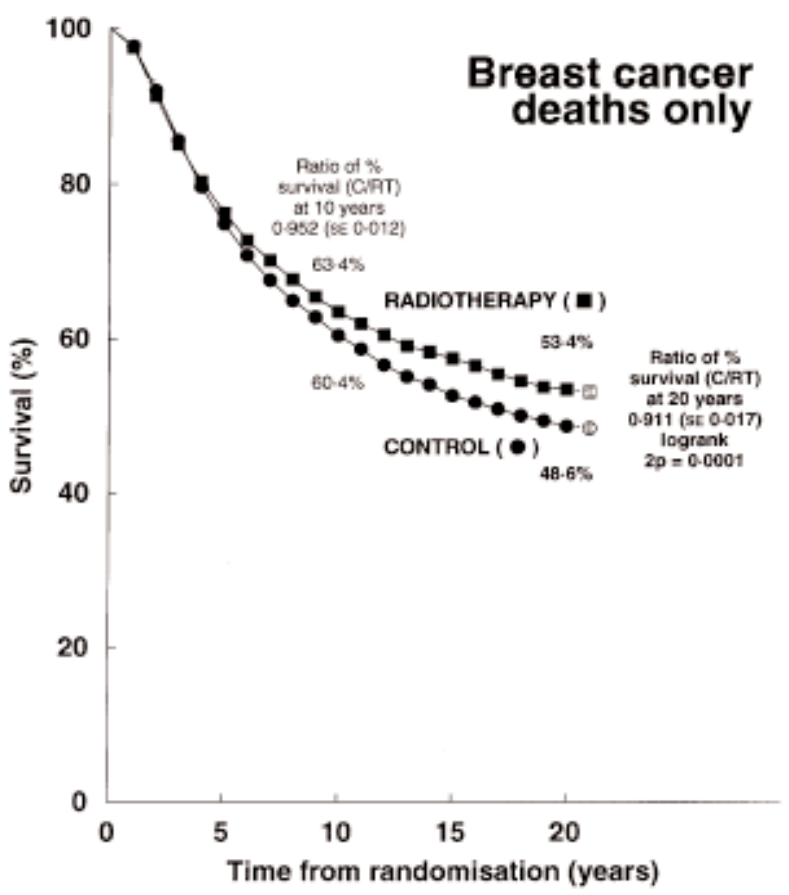

Fig. 5. EBCTCG 2000.

10\% la incidencia de recidivas a 20 años (Fig. 4). Sin embargo, el impacto dentro de la sobrevida global está sin demostrar. Los tratamientos con o sin RT no se diferencian en 


\section{H. Marsiglia y cols.}

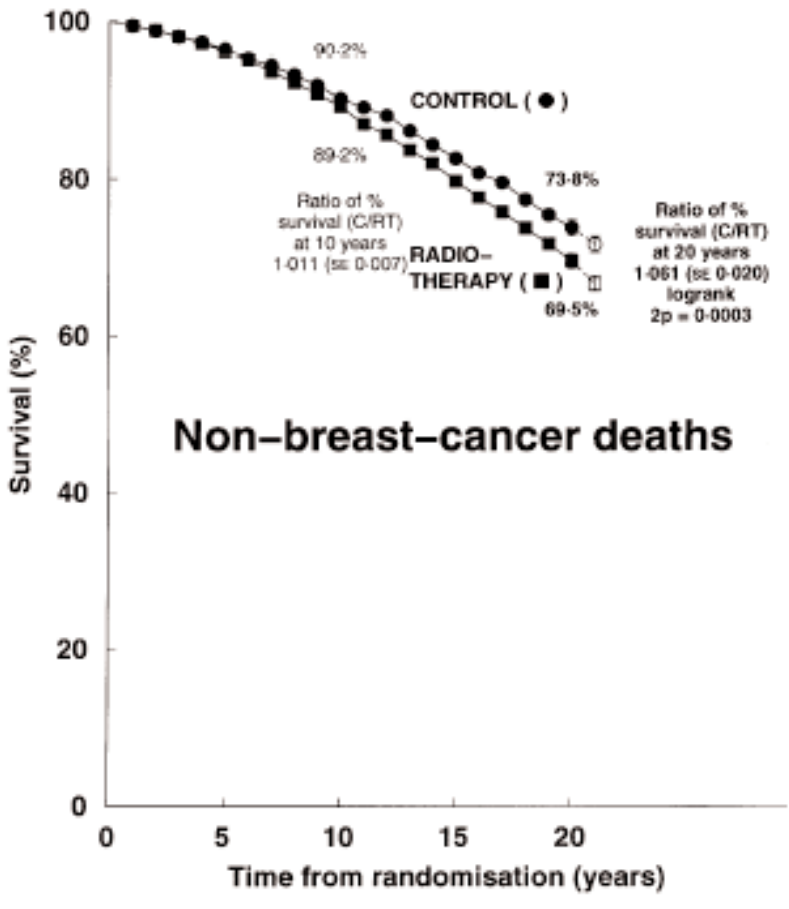

Fig. 6. EBCTCG 2000.

términos de sobrevida, cualquiera sea su status ganglionar. Por otro lado, un análisis sobre la sobrevida específica de decesos por cáncer, insinúa un beneficio de la RT, aumentando de un 48.6 a un $53.4 \%$, la sobrevida (Fig. 5), sin incidencia en la sobrevida global de decesos por enfermedades cardiovasculares intercurrentes (Fig. 6).

Los resultados remarcan el problema mayor, en la radiotoxicidad cardíaca y pulmonar (Cuzick 1994, Ruqvist 1992). En definitiva, no puede haber beneficios en la sobrevida global, si la técnica no es óptima.

\section{Perspectivas terapéuticas en el carcinoma infiltrante}

\section{Tratamiento conservador}

Para llevar a cabo un tratamiento conservador, es necesario seleccionar los pacientes en función de su edad, del estadio tumoral y de las características de las técnicas que se utilicen en el tratamiento (Rutgers 2001).

\section{Pacientes seleccionados para randomizar los beneficios o no de una RT externa:}

Son pacientes de la 3ra edad, T1, RH+, márgenes-, y tratamiento por tamoxifeno. Hasta el momento esto está avalado solamente por el Scottish Breast Cancer Group, que está en curso de aprobación (A randomised breast cancer trial to assess local control in older patients-post operative radiotherapy in minimum risk elderly).

\section{Pacientes seleccionados para una RT hipo-fraccionada en el Cancer de Mama:}

Son pacientes también mayores a 65 años, con una tumorectomía-curetaje axilar, una irradiación post-operatoria de 50 Gy en 5 semanas sobre el conjunto de la glándula mamaria (Vlastos 2001, Solin 1995). La única contra con estos pacientes, es que su movilidad se encuentra a veces reducida, no pudiendo cumplir el tratamiento (Ballard-Barbash 1996, Hebert-Croteau 1999, Mandelblatt 2000). Por tratar de mejorar esta terapéutica, ya hay de trials en fase II, intentando el mejor esquema de hiperfraccionamiento, y menos tóxico, posible (Hannoun-Levi, Marsiglia 2003 in press).

\section{Pacientes seleccionados (>65años, T1, NO,}

con Carcinoma exlcusivamente infiltrante o canalicular, sin multifocalidad ni extensión intraductal), para irradiación parcial de mama

El concepto de irradiación parcial de mama se basa en hacer una irradiación hiperfraccionada de manera acelerada, dando una dósis "biológicamente equivalente" a 50 Gy en 5 sem. de irradiación convencional, pero en un tiempo más corto (entre 1 y 5 días). La radioterapia externa perioperatoria (IORT) (Veronesi 2001, Vaidya 2001), y la curiterapia a bajo débito de dósis (LDR) (Vicini 1999-2001, King 2000, Krishnan 2001) o alto débito de dósis (HDR) (Vicini 2002, Wazer 2002, Polgar 2002) fueron el objeto de numerosos estudios en fase II, con resultados satisfactorios en el plano estético y en el control local optimal.

Muchos trials en fase III estan en curso para poner en evidencia la ausencia de una diferencia significativa entre control local e irradiación parcial de mama y una irradiación convencional tomando en cuenta la totalidad de la glándula mamaria. Esta es una de las estrategias de elección para las mujeres de la tercera edad. Esta técnica todavía, necesita ser evaluada en terminos de falsabilidad y reproductibilidad en diferentes centros (Marsiglia 2003, Bulletin du Cancer in press). En el contexto de un protocolo, la Federation Nationale de Centres de Lutte Contre le Cancer (FNCLCC, PAC GERICO 03, Hannoun-Levi, Marsiglia) viene de estar aprobada, y las inclusiones seran efectivas a partir del 2003: "Tratamiento radio-quirúrgico del cáncer de mama en la mujer mayor de 65 años, sin invasión ganglionar: estudios de fase II multicéntrico de la falsabilidad y reproductividad de una radioterapia concentrada y focalizada" (con un estudio médico-ecónomico).

\section{Conclusión}

Existen numerosos estudios hechos a lo largo de los últimos 20 años, que demuestran la eficacia de la Radioterapia adyuvante en mama, en comparación con la cirugía como terapia única. La tasa de recidiva local de la asociación radio-cirugía puede bajar un $30 \%$ el riesgo relativo de recidiva loco-regional. Este efecto benéfico se ha mantenido a lo largo del tiempo, como lo muestran los últimos trials randomizados, y los meta-análisis publicados entre 1995 y 2000. Aunque no haya sido estudiada directamente la sobrevida global, podemos insinuar a partir de las conclusiones de los últimos meta-análisis, una prolongación de la sobrevida por 
cáncer. Por otra parte, el aumento potencial a largo plazo de muerte por enfermedad intercurrente (sobre todo las cardiovasculares) nos lleva a efectuar técnicas rigurosas que no sobreexpongan al paciente a una toxicidad cardíaca o pulmonar. Las nuevas técnicas de irradiación en curso de evaluación, que incluyen la intensidad modulada, y la simulación por TAC, permitirán mejorar aún más los resultados terapéuticos. Por último, acompañando estas innovaciones técnicas, las estrategias terapéuticas que tienden a irradiar exclusivamente el lecho tumoral (irradiación parcial de mama) se estan comenzando a utilizar con mucho éxito, continuando el panorama prometedor, para este tipo de patología.

\section{Bibliografía}

1. Arriagada R, Le MG, Rochard F, Contesso. Conservative treatment versus mastectomy in early breast cancer: patterns of failure with 15 years of follow-up data. Institut Gustave-Roussy Breast Cancer Group. J Clin Oncol 1996;14(5):1558-64.

2. Arriagada R, Mouriesse H, Sarrazin D, Clark RM, Deboer G. Radiotherapy alone in breast cancer. I. Analysis of tumor parameters, tumor dose and local control: the experience of the Gustave-Roussy Institute and the Princess Margaret Hospital. Int J Radiat Oncol Biol Phys 1985 Oct; 11(10):1751-7.

3. Arriagada R, Rutqvist LE, Mattsson A, Kramar A, Rotstein S. Adequate locoregional treatment for early breast cancer may prevent secondary dissemination. J Clin Oncol $1995 \mathrm{Dec}$; 13(12):2869-78.

4. Arnulf G. Lymphoedema of the upper limb after Halsted's operation of radical mastectomie. Lymphography and phlebography. Therapeutic consequences. Vasc Surg 1973; $7(1): 36$ 44.

5. Auquier A, Rutqvist LE, Host $\mathrm{H}$, et al. Post-mastectomy megavoltage radiotherapy: The Oslo and Stockholm trials. Eur J Cancer 1992; 28:433-7.

6. Balduci L, Lyman GH. Cancer in the elderly.Epidemiologie and clinical implications.Clin Geriatr Med 1997; 13(1):1-14.

7. Ballard-Barbash R, Potosky AL, Harlan LC, Nayfield SG, Kessler LG. Factors associated with surgical and radiation therapy for early stage breast cancer in older women. J Natl Cancer Inst 1996; 88(11):716-26.

8. Bartelink $\mathrm{H}$, Horiot JC, Poortmans $\mathrm{P}$, Struikmans $\mathrm{H}$, Van den Bogaert W, Barillot I, Fourquet A, Borger J, Jager J, Hoogenraad W, Collette L, Pierart M; European Organization for Research and Treatment of Cancer Radiotherapy and Breast Cancer Groups. Recurrence rates after treatment of breast cancer with standard radiotherapy with or without additional radiation. $\mathrm{N}$ Engl J Med 2001 Nov 8; 345(19):1378-87.

9. Bijker N, Peterse JL, Duchateau L, et al Risk factor for recurrence and metastasis after breast conserving therapy for ductal carcinoma in situ: Analysis of European Organisation for Research and Treatment of Cancer trial 10853. J Clin Oncol 2001; 19:2263-71

10. Brenier JL. The role of the Halsted operation in treatment of breast cancer. Int Surg 1967; 47(3):288-90.

11. Clark RM, Whelan T, Levine $M$, Roberts $R$, Willan $A, M c C u l l o c h$ $\mathrm{P}$, Lipa M, Wilkinson RH, Mahoney U. Randomized clinical trial of breast irradiation following lumpectomy and axillary dissection for node-negative breast cancer: an update. Ontario Clinical Oncology Group. J Natl Cancer Inst 1996; 88(22):165964.
12. Cunsolo A, Guarasci N, Maldarizzi F, Bragaglia R, Mattioli S. Treatment of cancer of the breast with Patey's modified radical mastectomy. Minerva Chir 1979; 34(7):549-52.

13. Cutuli B, Cohen-Solal-Le N, De Lafontan B, Mignotte $\mathrm{H}$ et al Breast-conserving therapy for ductal carcinoma in situ of the breast: the french cancer centers' experience. Int J Radiat Oncol Biol Phys 2002; 53(4):868-79.

14. Cuzick J, Stewart H, Rutqvist L, Houghton J, Edwards R, Redmond C, Peto R, Baum M, Fisher B, Host H, et al. Cause-specific mortality in long-term survivors of breast cancer who participated in trials of radiotherapy. J Clin Oncol 1994 Mar; 12(3):447-53.

15. Chardot C, Adolff-O'Reilly A. Present sequelae and psychological repercussions of Halsted's operation. A detailed survey of 95 patients. Presse Med 1968; 76(38):1813-6.

16. De Vathaire F, Kscielny S, Rezvani A, Laplanche A, Estève J, Ferlay J. Réseau FRANCIM, Ed. statistiques de santé. Estimation de l'incidence des cancers en France 1983-1987. Paris: Los éditions INSERM; 1996.

17. Early Breast Cancer Trialists' Collaborative Group. Effects of radiotherapy and surgery in early breast cancer. An overview of the randomized trials. N Engl J Med 1995 Nov 30; 333(22): 1444-55.

18. Early Breast Cancer Trialists Collaborative Group. Favourable and unfavourable effects on long-term survival of radiotherapy for early breast cancer: an overview of the randomised trials. The Lancet 2000; 355:1757-70.

19. De Vita VT, Hellman S and Rosemberg S. Cancer Principlos \& Practice of Oncology. Lippincott Williams \& Wilkins. 6th edition, 2001.

20. Fisher B, Redmond C, Poisson R, Margolose R, Wolmark N, Wickerham L, Fisher E, Deutsch M, Caplan R, Pilch $Y$, et al. Eight-year results of a randomized clinical trial comparing total mastectomy and lumpectomy with or without irradiation in the treatment of breast cancer. N Engl J Med 1989 30; 320:822-8.

21. Fisher $B$, Anderson $S$, Fisher $E$, et al. Significance of ipsilateral breast tumor recurrence after lumpectomy. Lancet 1991; 338:327-31

22. Fisher B, Constantino J, Redmond C, Fisher E, Margolose R, Dimitrov N, Wolmark N, Wickerham DL, Deutsch M, Ore L, Mamounas E, Poller W, Kavanah M. Lumpectomy compared with lumpectomy and radiation therapy for the treatment of intraductal breast cancer. N Engl J Med 1993; 328:1581-6.

23. Fisher B, Anderson S, Redmond CK, Wolmark N, Wickerham DL, Cronin WM. Reanalysis and Results after 12 Years of Follow-up in a Randomized Clinical Trial Comparing Total Mastectomy with Lumpectomy with or without Irradiation in the Treatment of Breast Cancer. N. Engl J Med 1995; 333:1456-61.

24. Fisher B, Land S, Mamounas E, et al. Prevention of invasive breast cancer in women with ductal carcinoma in situ: An update of the national surgical adjuvant breast and bowel porject experience. Semin Oncol 2001; 28:400-18.

25. Fisher B, Anderson S, Bryant J, Margolose RG, Deutsch M, Fisher ER, Jeong JH, Wolmark N. Twenty-year follow-up of a randomized trial comparing total mastectomy, lumpectomy, and lumpectomy plus irradiation for the treatment of invasive breast cancer. N Engl J Med 2002 Oct 17; 347(16):1233-41.

26. Fletcher $\mathrm{GH}, \mathrm{McN}$ eese $M D$, Oswald MJ Long-range results for breast cancer patients treated by radical mastectomy and postoperative radiation without adjuvant chemotherapy: un update. Int J Radiat Oncol Biol Phys 1989; 17:1 1-4.

27. Fortin A, Karochelle M, Laverdiere J, Lavertu S, Tremblay D Lo- 


\section{H. Marsiglia y cols.}

cal failure is responsible for the decrease in survival for patients with breast cancer treated with conservative surgery and postoperative radiotherapy. J Clin Oncol 1999; 17:101-9.

28. Fourquet A, Campana F, Mosseri V, et al. Iridium-192 versus cobalt 60 boost in 3-7 cm breast cancer treated by irradiation alone: final results of a randomized trial. Radiother Oncol 1995; 34:114-20.

29. Fowble B, Gray R, Gilchrist K et al. Identification of a subgroup of patients with breast cancer and histologically positive axillary nodes receiving adjuvant chemotherapy who may benefit from postoperative radiotherapy. J Clin Oncol 1988; 6:1 107-17.

30. GLOBOCAN 2000: Cancer Incidence, Mortality and Prevalence Worldwidee, Version 1.0 IARC Cancer Base N5. Lyon, IARC Press, 2001.

31. Griem KL, Henderson IC, Gelman R, et al. The 5-years results of a randomized trial of adjuvant radiation therapy after chemotherapy in breast cancer patients treated with mastectomy. J Clin Oncol 1987; 5:1546-55.

32. Haffty $B$, Reiss $M$, Beinfield $M$, et al. Ipsilateral breast tumor recurrences as predictor of distant disease: implications for systemic therapy at the time of local relapse. J Clin Oncol 1996; 14:52-7.

33. Hebert-Croteau N, Brisson J, Latreille J, Blanchette C, Deschenes $L$. Compliance with consensus recommendations for the treatment of early stage breast carcinoma in elderly women. Cancer 1999; 85(5): 1104-13.

34. Helinto $M$, Blomqvist $C$, Heikkila $P$, et al. Post-mastectomy radiotherapy in PT3NOMO breast cancer: is it neaded? [see coments]. Radiother Oncol 1999; 52:213-7.

35. Holland $\mathrm{R}$, Solke $\mathrm{HJ}$, Veling $M$ et al. Histologic multifocality of Tis, T1, T2 breast carcinoma. Implications for clinical trials of breast-conserving surgery. Cancer 1985; 56:979-90.

36. Jacobson JA, Danforth DN, Cowan KH, d'Angelo T, Steinberg SM, Pierce L, Lippman ME, Lichter AS, Glatstein E, Okunieff P. Ten-year results of a comparison of conservation with mastectomy in the treatment of stage I and II breast cancer. N Engl J Med 1995; 332(14):907-11.

37. Koscielny S, Tubiana M. The link between local recurrence and distant metastases in human breast cancer. Int J Radiat Oncol Biol Phys. 1999; 43:11

38. Krawczyk JJ, Engel B. The importance of surgical clips for adequate tangential beam planning in breast conserving surgery and irradiation. Int J Radiat Oncol Biol Phys 1999 Jan 15; 43(2):347-50.

39. Kurtz J for the EUSOMA Working Party. The curative role of radiotherapy in the treatment of operable breast cancer. Eur J Cancer 2002; 38:1961-74.

40. Hannoun-Lévi JM, Marsiglia $\mathrm{H}$, et al. Radiothérapie hypofractionnée dans le cancer du sein. Bulletin du cancer 2003.

41. King TA, Bolton JS, Kuske RR, Fuhrman GM, Scroggins TG Jiang XZ. Long-term results of wide-field brachytherapy as the sole method of radiation therapy after segmental mastectomy for $T$ (is, 1, 2) breast cancer. Am J Surg. 2000; 180(4):299304.

42. Krishnan L, Jewell WR, Tawfik OW, Krishnan EC. Breast conservation therapy with tumor bed irradiation alone in a selected group of patients with stage I breast cancer. Breast J 2001; 7(2):91-6.

43. Liljegren G, Holmberg L, Bergh J, Lindgren A, Tabar L, Nordgren $\mathrm{H}$, Adami $\mathrm{HO}$. 10-Year results after sector resection with or without postoperative radiotherapy for stage I breast cancer: A randomized trial.J Clin Oncol 1999; 17(8):2326.
44. Maddox WA, Carpenter JT Jr, Laws HL, Soong SJ, Cloud G, Urist MM, Balch CM. A randomized prospective trial of radical (Halsted) mastectomy versus modified radical mastectomy in 311 breast cancer patients. Ann Surg 1983; 198(2):207-12.

45. Maher M; Campana F; Mosseri V; Dreyfus H; Vilcoq JR; Gautier C; Asselain B; Fourquet A. Breast cancer in elderly women: a retrospective analysis of combined treatment with tamoxifen and once-weekly irradiation. Int J Radiat Oncol Biol Phys 1995 Feb 15; 31(4):783-9.

46. Mandelblatt JS, Hadley J, Kerner JF, Schulman KA, Gold K, Dunmore-Griffith J, Edge S, Guadagnoli E, Lynch JJ, Meropol NJ, Weeks JC, Winn R. Patterns of breast carcinoma treatment in older women: patient preference and clinical and physical influences. Cancer 2000; 89(3):561-73.

47. Marsiglia $\mathrm{H}$, Hannoun-Levi $J M$ et al. Irradiation partielle du sein: pourquoi, comment? Cancer Radiothérapie 2003.

48. Morris AD, Morris RD, Wilson JF, et al. Breast-conserving therapy vs mastectomy in early-stage breast cancer: a meta-analysis of 10-years survival. Cancer J Sci Am 1997; 3:6-12.

49. Msss, Fichiers des tumores, 1984-1995. Direction de la santé publique de Montréal-Centre. Mise à jour septembre 2000.

50. NIH consensus conference. Treatment of early-stage breast cancer. JAMA 1991; 265:391-5.

51. Nemoto T, Dao TL. Is modified radical mastectomy adequate for axillary lymph node dissection? Ann Surg 1975; 182(6):722-3.

52. Overgaard $M$, Hansen PS, Overgaard J, Rose $C$, Andersson $M$, Bach F, Kjaer M, Gadeberg CC, Mouridsen HT, Jensen MB, Zedeler K. Postoperative radiotherapy in high-risk premenopausal women with breast cancer who receive adjuvant chemotherapy. Danish Breast Cancer Cooperative Group 82b Trial. N Engl J Med 1997 Oct 2; 337(14):949-55.

53. Overgaard $M$, Jensen $M B$, Overgaard J, Hansen PS, Rose $C$, Andersson $M$, Kamby $C$, Kiaer M, Gadeberg CC, Rasmussen $B B$, Blichert-Toft M, Mouridsen HT. Postoperative radiotherapy in high-risk postmenopausal breast-cancer patients given adjuvant tamoxifen: Danish Breast Cancer Cooperative Group DBCG 82c randomized trial. Lancet 1999 May 15; 353(9165):1641-8.

54. Polgar C, Sulyok Z, Fodor J et al. Sole brachytherapy of the tumor bed after conservative surgery for $\mathrm{Tl}$ breast cancer: five years results of a phase I-II study and initial findings of a randomized phase III trial. J Surg Oncol 2002; 80(3):121-8.

55. Ragaz J, Jackson SM, Le N, Plenderleith $I H$, Spinelli JJ, Basco VE, Wilson KS, Knowling MA, Coppin CM, Paradis M, Coldman AJ, Olivotto IA. Adjuvant radiotherapy and chemotherapy in node-positive premenopausal women with breast cancer. $\mathrm{N}$ Engl J Med 1997 Oct 2; 337(14):956-62.

56. Ravitch MM. Carcinoma of the breast: the place of the Halsted radical mastectomy. Johns Hopkins Med J 1971; 129(4):20211.

57. Remouchamps V, Vicini F, Sharpe M, Kestin L, Martinez A, Wong J. Significant reductions in heart and lung doses using deep inspiration breath hold with active brathing control and intensity-modulated radiation therapy for patients treated with locoregional breast irradiation. Int J Radiat Oncol Biol Phys 2003; 55(2):392-406.

58. Romestaing $P$, Lehingue $Y$, Carrie $C$, Coquard $R$, Montbarbon $X$, Ardiet JM, Mamelle N, Gerard JP. Role of a 10-Gy boost in the conservative treatment of early breast cancer: results of a randomized clinical trial in Lyon, France. J Clin Oncol 1997; 15(3):963-8.

59. Rutgers EJ, EUSOMA Consensus Group. Quality control in the 
locoregional treatment of breast cancer. Eur J Cancer 2001 Mar; 37(4):447-53.

60. Rutqvist LE, Lax I, Fornander T, Johansson H. Cardiovascular mortality in a randomized trial of adjuvant radiation therapy versus surgery alone in primary breast cancer. Int J Radiat Oncol Biol Phys 1992; 22(5):887-96.

61. Saucr R, Tulusan A, Lang $N$ et al. Can breast irradiation be omitted in low-risk breast cancer patients after segmentectomy? First results of the Erlangen protocol. Int J Radiat Oncol Biol Phys 1993; 27 (Suppl 1):146 (abstract).

62. Schnitt SJ, Hayman J, Gelman R et al. A prospective study of conservative surgery alone in the treatment of selected patients with stage 1 breast cancer. Cancer 1996; 77:1094-100.

63. Solin $\amalg$, Schultz DJ, Fowble BL. Ten-year results of the treatment of early-stage breast carcinoma in elderly women using breastconserving surgery and definitive breast irradiationirradiation. Int J Radiat Oncol Biol Phys. 1995; 33(1):45-51

64. Stefanik D, Goldberg R, Byrne P, et al. Local-regional failure in patients treated with adjuvant chemotherapy for breast cancer. $\mathrm{J}$ Clin Oncol 1985; 3:660-5.

65. Vaidya JS, Baum M, Tobias JS, D'Souza DP, Naidu SV, Morgan S, Metaxas M, Harte KJ, Sliski AP, Thomson E. Targeted intra-operative radiotherapy (Targit): an innovative method of treatment for early breast cancer. Ann Oncol 2001 Aug; 12(8):1075-80.

66. Van de Steene J, Soete G, Storme G. Adjuvant radiotherapy for breast cancer significantly improves overall survival: the missing link. Radiother Oncol 2000; 55:263-72.

67. van Dongen JA, Voogd AC, Fentiman IS, Legrand C, Sylvester RJ, Tong D, van der Schueren E, Helle PA, van Zijl K, Bartelink $\mathrm{H}$. Long-term results of a randomized trial comparing breastconserving therapy with mastectomy: European Organization for Research and Treatment of Cancer 10801 trial. J Natl Cancer Inst 2000; 92(14):1143-50.

68. Veronesi $U$, et al. Comparing radical mastectomy with quadrantectomy, axillary dissection, and radiotherapy in patients with small cancers of the breast. N Engl J Med 1981; 305(1):611.

69. Veronesi U, Cascinelli N, Mariani L, Greco M, Saccozzi R, Luini A, Aguilar M, Marubini E. Twenty-year follow-up of a randomized study comparing breast-conserving surgery with radical mastectomy for early breast cancer. N Engl J Med. 2002 Oct 17; 347(16): 1227-32.

70. Veronesi U, Marubini E, Mariani L, Galimberti V, Luini A, Veronesi P, Salvadori B, Zucali R. Radiotherapy after breast-conser- ving surgery in small breast carcinoma: long-term results of a randomized trial. Ann Oncol 2001; 12(7):997-1003.

71. Vicini $F$, Kini VR, Chen $P$, Horwitz $E$, Gustafson $G$, Benitez $P$, Edmundson G, Goldstein N, McCarthy K, Martínez A. Irradiationlrradiation of the tumor bed alone after lumpectomy in selected patients with early-stage breast cancer treated with breast conserving therapy. J Surg Oncol 1999; 70(1):33-40.

72. Vicini FA, Baglan KL, Kestin LL, Mitchell C, Chen PY, Frazier RC, Edmundson G, Goldstein NS, Benitez P, Huang RR, Martínez A. Accelerated treatment of breast cancer. J Clin Oncol 2001; 19(7): 1993-2001.

73. Vicini F, Baglan K, Kestin L, Chen P, Edmundson G, Martínez A. The emerging role of brachytherapy in the management of patients with breast cancer. Semin Radiat Oncol 2002 Jan; 12(1):31-9.

74. Vlastos G, Mirza NQ, Meric F, Hunt KK, Kuerer HM, Ames FC, Ross MI, Buchholz TA, Hortobagyi GN, Singletary SE. Breast conservation therapy as a treatment option for the elderly. The M. D. Anderson experience. Cancer 2001; 92(5):1092-100.

75. Voogd AC, Nielsen M, Peterse JL, Blichert-Toft M, Bartelink $H$, Overgaard M, van Tienhoven G, Andersen KW, Sylvester RJ, van Dongen JA; Danish Breast Cancer Cooperative Group. Breast Cancer Cooperative Group of the European Organization for Research and Treatment of Cancer. Differences in risk factors for local and distant recurrence after breast-conserving therapy or mastectomy for stage I and II breast cancer: pooled results of two large European randomized trials. J Clin Oncol 2001 Mar 15; 19(6):1688-97 [Erratum in: J Clin Oncol 2001 May 1; 19(9):2583].

76. Vrieling $C$, Collette $L$, Fourquet $A$, et al. The influence of the boost in breast-conserving therapy on cosmetic outcome in the EORTC \&quot; boost versus non boost\&quot; trial. EORTC Radiotherapy and breast cancer cooperative groups. European organization for research and treatment of cancer. Int J Radiat Oncol Biol Phys 1999; 45:677-85.

77. Wazer DE, Berle L, Graham R, Chung M et al. Preliminary results of a phase I/II study of HDR brachytherapy alone for T1/T2 breast cancer. Int J Radiat Oncol Biol Phys 2002; 53(4):889-97.

78. Whelan TJ, Julian J, Wright J, et al. Does locoregional radiation therapy improve survival in breast cancer? A meta-analysis. J Clin Oncol 2000; 18:1220-9.

79. Winchester DJ, Menck HR, Winchester DP. The national cancer data base report on the results of a large nonrandomized comparison of breast preservation and modified radical mastectomy. Cancer 1997; 80:162-7. 